\title{
Enzymatic Glucose Biosensor of Buckypaper: an engineered surface of CNT
}

Much recent researches have focused on electrochemical biosensor $\mathrm{s}$ to meet the growing demands for rapid and accurate diagnosis tests for disease. Conventional enzyme-based sensors propose favorably selective and sensitive determination of glucose at the outlay of low stability. Thus, promoting the comfortable, sensitive, rapid and consistent strategies has an impressive role for determining the human glucose level. Here, we synthesis a novel macro scale network of carbon nanotubes "Buckypaper" by a non-ionic surfactant (Tween 20). Buckypaper (BP) is a plate-like carbon nanotube (CNT) films obtained from single-walled CNTs (SWCNTs) in a form of entangled networks of ropes and bundles of CNT held together by van der Waals. The BP was characterized by Fourier transform infrared spectroscopy (FTIR) and Field emission scanning electron microscopy (FESEM). The electrochemical behavior and electrical conductivity of the BP was investigated through cyclic voltammetry (CV) and electrochemical impedance spectroscopy (EIS).BP shows a significant electrochemical result compared with the Carbon nanotube and Graphene. The BP was utilized as a surface for glucose detection with a linear range of $0.1 \times 10^{-} 3-$ $13 \times 10-3 \mathrm{M}$ and the electrochemical response of the interfering species was also examined and welldefined glucose response was obtained and insignificant response were observed for interfering species. The sensor exhibited excellent performance for glucose at applied potential of $0.575 \mathrm{~V}$. To the best of our knowledge, on the first researches on the Buckypaper for biosesning application as an individual surface for enzyme stabilization and enzymatic sensing without functionalization.

Keywords: Buckypaper, Biosensor, Elechtrochemistry

\section{Introduction}

Glucose is named as bio and green fuel in human's, animal's and plant's body cell. It's on of most useful and important materials in nature but even this useful material must controlled in dosage[1]. High and not usage dosage if glucose can cause diseases. There was many biosensors build over the years for monitoring the glucose in human's and other creature's body. One the most favorable of biosensors of new researches is multiple type of carbon Nano tubes[2]. The high conductivity of carbon nanotubes has led to use these nanostructures in many different applications such as biosensors[3]. Single-walled carbon nanotubes (SWCNTs) are formed of just one layer of graphite and has very narrow size distribution, whereas multi-walled carbon nanotubes (MWCNTs) are formed of more than two layers of graphite[4]. The nanoscale of structure makes carbon nanotube impractical to be used in industrial activity individually, so some methods have been developed to fabricate macro scale networks of carbon nanotubes in a plate form[5]. Buckypaper is plate-like CNT-films obtained from SWCNTs and MWCNTs in a form of entangled networks of ropes and bundles of CNT held together by Van der Waals interactions[6]. There are two common methods which BP can be made from: 1-Domino pushing; a dry and in situ method for the preparation of well aligned CNTs in the BP, [7] and 2Dispersion and Filtration; the low cost and useful method.[8] To achieve good tube dispersion in 
the latter method, surfactants are used. The rule of the surfactant is to break bundles and to well disperse of the tubes leading to stabilization of suspension and better quality of the BP. There are some kinds of non-ionic surfactants such as Triton X-100, Span 20, Polysorbate 20 (Tween 20), Polysorbate 80 (Tween 80) and Saponin. The last one, Saponin, is a natural surfactant that is extracted from various plant species. The structures of the surfactants are shown in Figure 1. The residual of surfactant in this method will decrease interface bonding and electrical properties of $\mathrm{BP}$, therefore, it has to be completely removed via washing with plenty of water[9]. BPs are utilized to solve low tube loading, lack of alignment and uncontrolled problems for nanocomposites fabrication[10] Due to the intrinsic properties, they could be used as electrode for batteries[7], and sensors[11].here we focus on biosensor usage. Investigation of the magnetic effect on CNT ropes alignment in mechanical properties of BPs[12] and the effects of surfactants and alignment on the physical properties of SWCNT BPs[13]has been previously described. Therefore, we decided to investigate the effect of the type of some non-ionic surfactants on electrochemical behavior of the prepared BPs. A BP made from $\mathrm{NH}_{2}$-functionilized multi-wall carbon nanotubes was also prepared in a similar manner using Triton X-100 and the effect of $\mathrm{NH}_{2}$ functional group on dispersion and electron transfer resistance was investigated. Increase in rate of electron transfer is favorable properties for biosensor act.<smiles>[2H][C]([CH])OCC(C)Oc1ccc(C(C)(C)CC(C)(C)C)cc1</smiles>
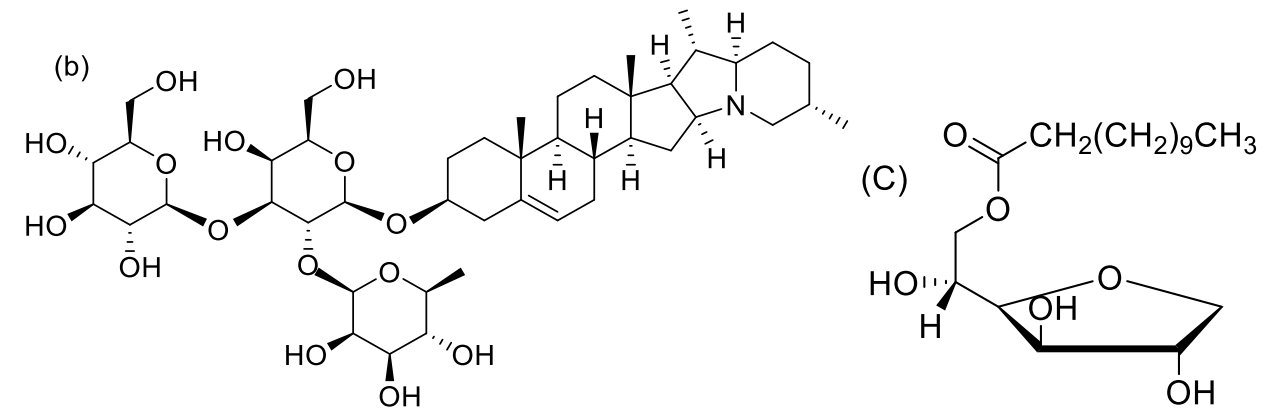


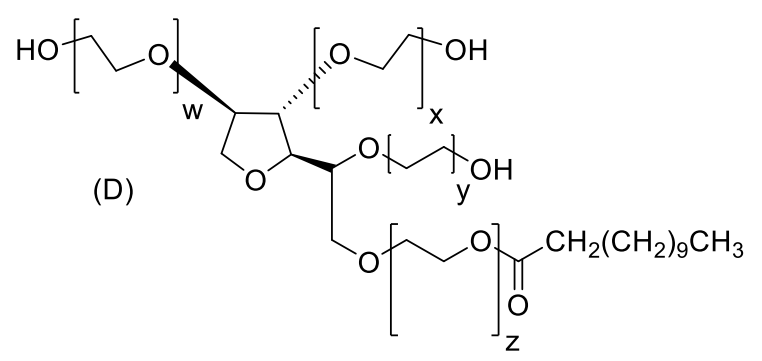

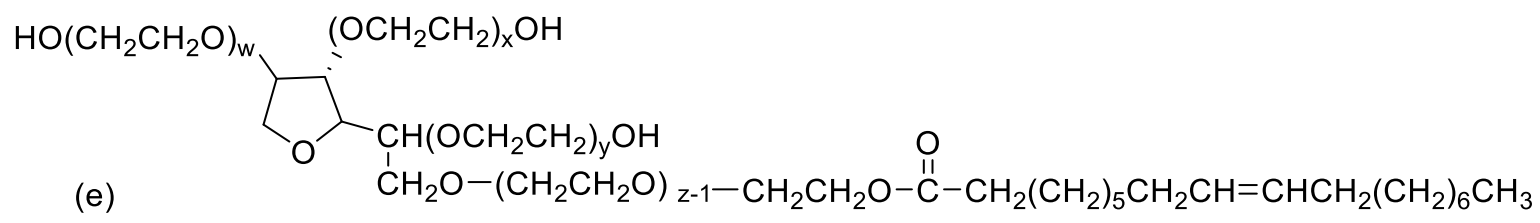

Fig1. Structure of: (a) Triton X-100, (b) Saponin, (c)Span 20, (d) Tween 20, (e) Tween 80

\section{2: Experimental}

BPs were prepared by first adding $10 \mathrm{mg}$ SWCNT and definite amount of nonionic surfactant according to their critical micelle concentration (CMC) in $50 \mathrm{ml}$ water. We used five different surfactant including: Tritx100, Tween20, Tween80, Span20 and Saponin. At first $10 \mathrm{mg}$ SWCNT and definite amount of the nonionic surfactant Tween20 (0.06 Mm), -according to it critical micelle concentration (CMC) - were added to $50 \mathrm{ml}$ water. The above resulting mixtures were subjected in an ultrasonic bath for $30 \mathrm{~min}$ then were stirred for 24 hours. After that, the suspensions were filtered by vacuum filtration through a polytetrafluoroethylene (PTFE) membrane filter $(0.45 \mu \mathrm{m}$ pore size $)$ and left to dry in room temperature which can be shown in fig .2.

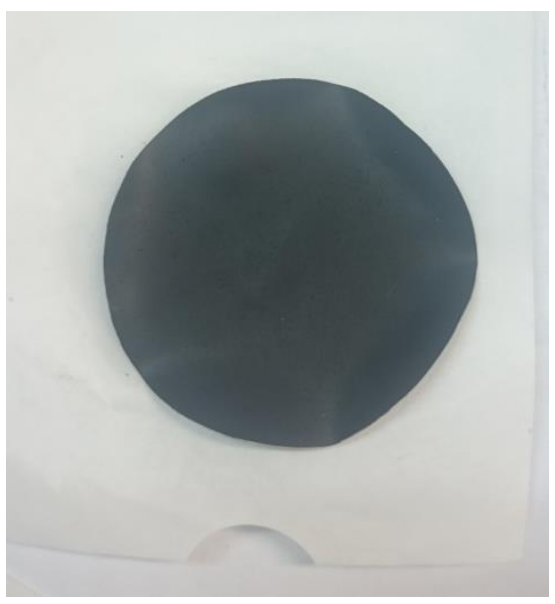

Fig.2. picture of synthesized BP 


\section{3: Result and discussion}

\subsection{Structure of characteristics}

Fig.3 displays (FESEM) of the SWCNTs arrangement in each BP. The FESEM images demonstrate that each of the BPs have different surface morphologies, according to the type surfactants prepared from and all the BPs were washed with plenty of water and isopropanol in a same condition. Fig.3a shows the morphology of BP/Tween 80 that demonstrate the surface uniformity with a few agglomerations. Fig 4b shows the FESEM micrograph of a BP/span 20.This image reveals a uniform morphology without any agglomeration and the distribution of CNTs bundles are individual and random, so the ropes integration can be observed, clearly. Figure 3d doesn't show any residual surfactant on the surface. The FESEM image of a BP/Tween 20 (Fig.3c) reveals high uniformity of surface similar to Span 20. Fig. 3d depicts BP synthesized from $\mathrm{NH}_{2}$-functionilized $\mathrm{CNT}$ with Triton X100. The bright spots are related to the position of $\mathrm{NH}_{2}$ agent groups at the end of CNTs, revealing less surface uniformity.

Based on Fig. 3e highly agglomeration is observed for the BP prepared from Saponin and the residual surfactant causes a self-entangled network with lack of surface uniformity. It's worth mentioning that the surfactant used in the procedure is remained on the BPs surface, leading to creation of disconnected sheet with great pores and reducing specific area caused low conductivity as the following sections. fig. $4 \mathrm{f}$ shows the BP which made by Triton $\mathrm{x} 100$ that is a conventional surfactant for preparing BPs used in each procedure for dispersing CNTs in aqueous condition. A surface with high packed CNTs agglomeration in some area can be observed for this BP, compared with BPs produced by Span 20 or Tween 20. 

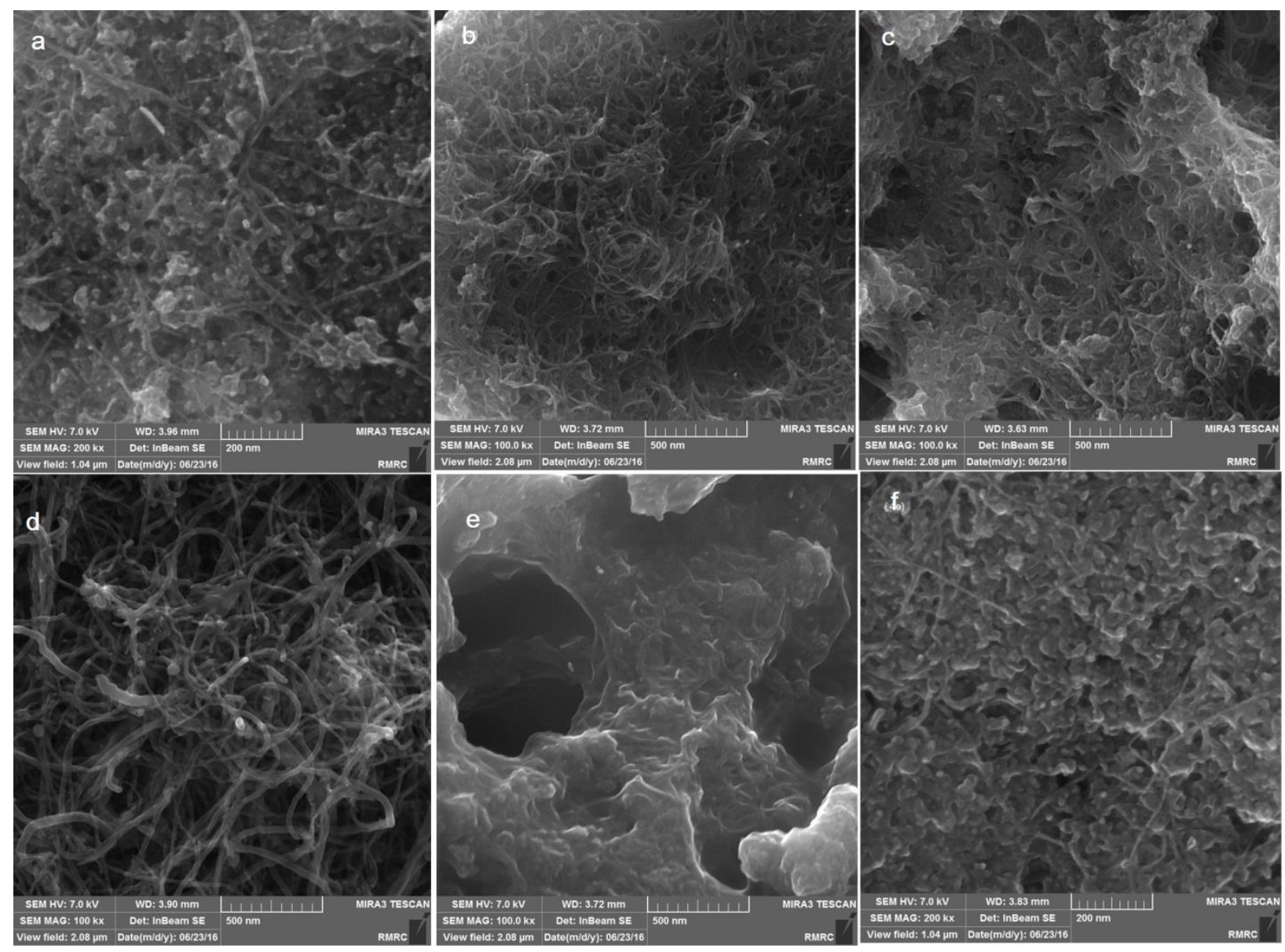

Fig .3 SEM images of BP by (a) Tween 80, (b) Span 20, (c) Triton X100, (d) NH2- Triton X-100, (c)Tween 20, (e) Saponin, (f) Triton X100

\subsection{Electrochemical Impedance Spectroscopy}

The impedance spectra consist of a semicircle portion and a liner portion. The semicircle diameter in higher frequencies is related to the electron-transfer resistance (Ret) and the liner part at lower frequencies is corresponded to diffusion process. The EIS of bare electrode shows $11000 \Omega$ that is much more than electrodes modified with different BPs. However, the least resistance was observed for the BP/Span 20 and Tween 20. It seems the surface uniformity without agglomeration can maybe help to fast electron transfer. Span 20 and Tween 20 demonstrated the least resistance and highest conductivity between the other BPs. BP/Saponin with shows the high resistance and undesirable electrochemical behavior Due to the presence of residual surfactant (Fig. 4). 


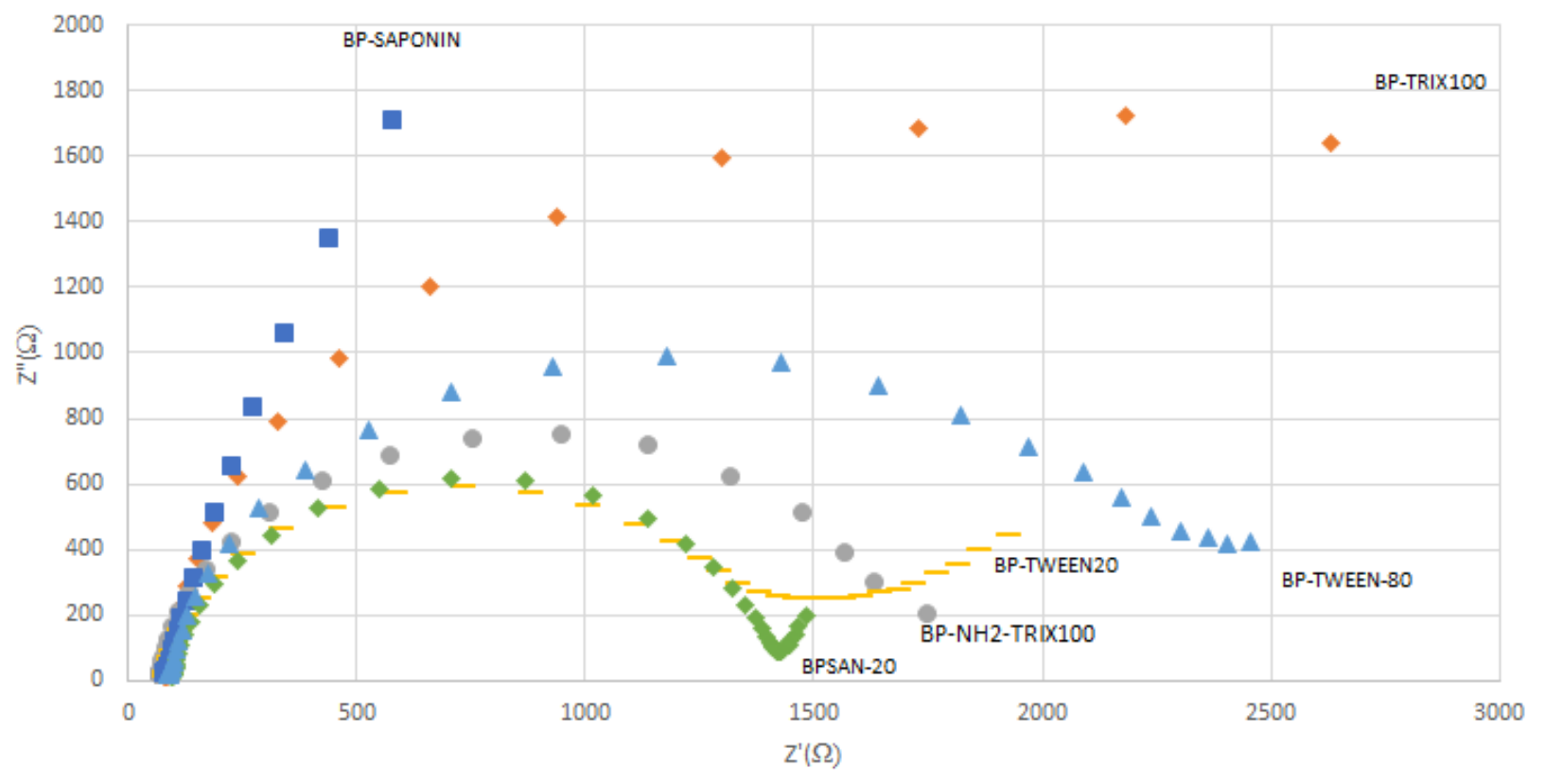

Fig.4 EIS of different obtained BPs /Span 20, Tween 20, Tween 80, Triton X-100, Saponin and BP$\mathrm{NH}_{2}$ /Triton $\mathrm{X} 100$ at $0.23 \mathrm{~V}$ with FTO as the working electrode and $\mathrm{Ag} / \mathrm{AgCl} / 3.0 \mathrm{M}$ as the reference electrode in electrochemical cell.

\subsection{Cyclic Voltammetry analysis}

CVs of the BP/Tween 20, BP/Span 20, and BP/Tween 80, BP/Tritonx100, BP/Saponin and BP$\mathrm{NH}_{2} / \operatorname{Trx} 100$ electrodes records in $0.1 \mathrm{M} \mathrm{K} 3 \mathrm{Fe}(\mathrm{CN})_{6}+0.1 \mathrm{M} \mathrm{PBS}(\mathrm{pH} 7.4)$ at a scan rate of 100 $\mathrm{mVs}^{-1}$. Fig.5 shows CVs of the electrode modified with different BPs that the same result can be observed as same as EIS. The well-defined oxidation and reduction peaks are observable due to the $\mathrm{Fe}^{3+} / \mathrm{Fe}^{2+}$ redox couple. All these results show that the BP/Span20 and BP/Tween20 significantly enhanced the electrical communication but, both (Ep) and peak current (Ip) slightly decreased in BP/Saponin and BP/Trx100, because of residual surfactant. 


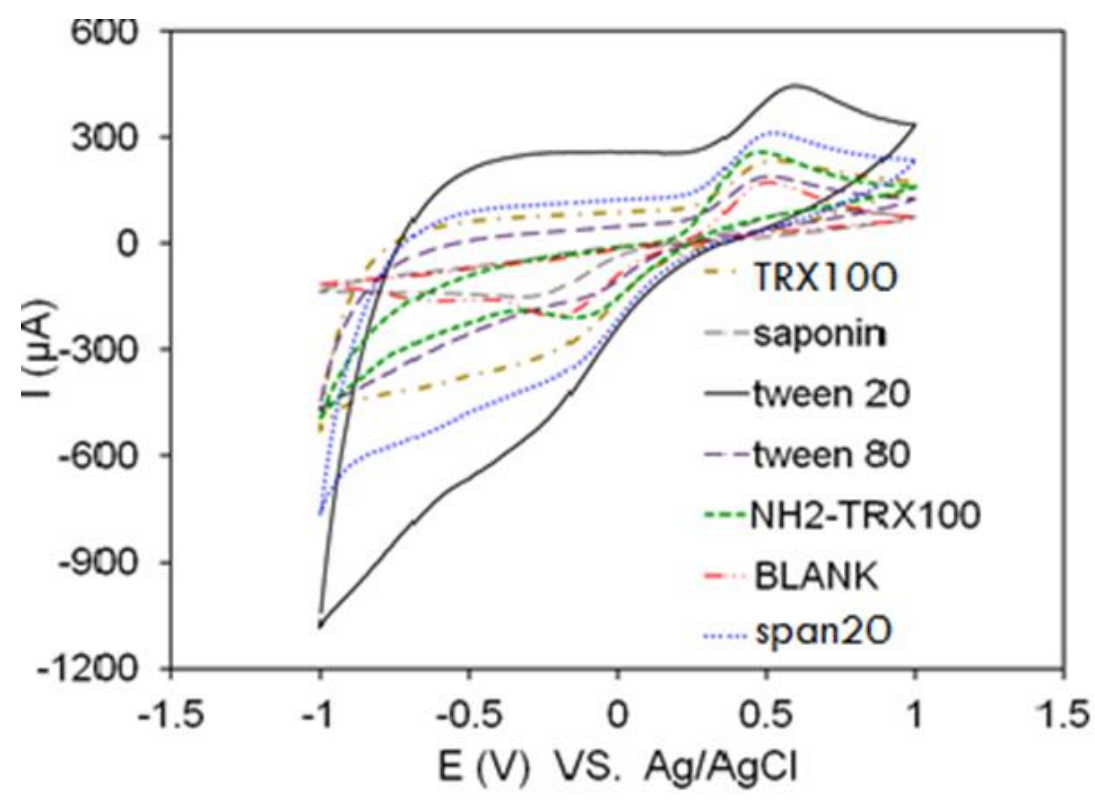

Fig.5. CVs of the modified electrodes by BPs /different surfactant in $1 \mathrm{mM} \mathrm{K}_{3}\left[\mathrm{Fe}(\mathrm{CN})_{6}\right]$ at $100 \mathrm{mVs}-1$

\subsection{Biosensing application}

Fig.6 shows the amperometric response of the BP (tween20)/FTO electrode examined with several additions of glucose spiked into PBS $0.05 \mathrm{M}$ solutions. The calibration curve for the glucose sensor is shown in the inset of Fig. 7 , which provides the regression equation, $\mathrm{I}(\mu \mathrm{A})=3.04 \mathrm{Cglucose}(\mu \mathrm{M})+$ 1.138 , with correlation coefficient of $\mathrm{R}^{2}=0.976$. The electrode has a linear concentration range of $0.1 \times 10^{-3}-12 \times 10^{-3} \mathrm{M}$ that can be comparable to the carbon base materials such as CNT and Graphen.

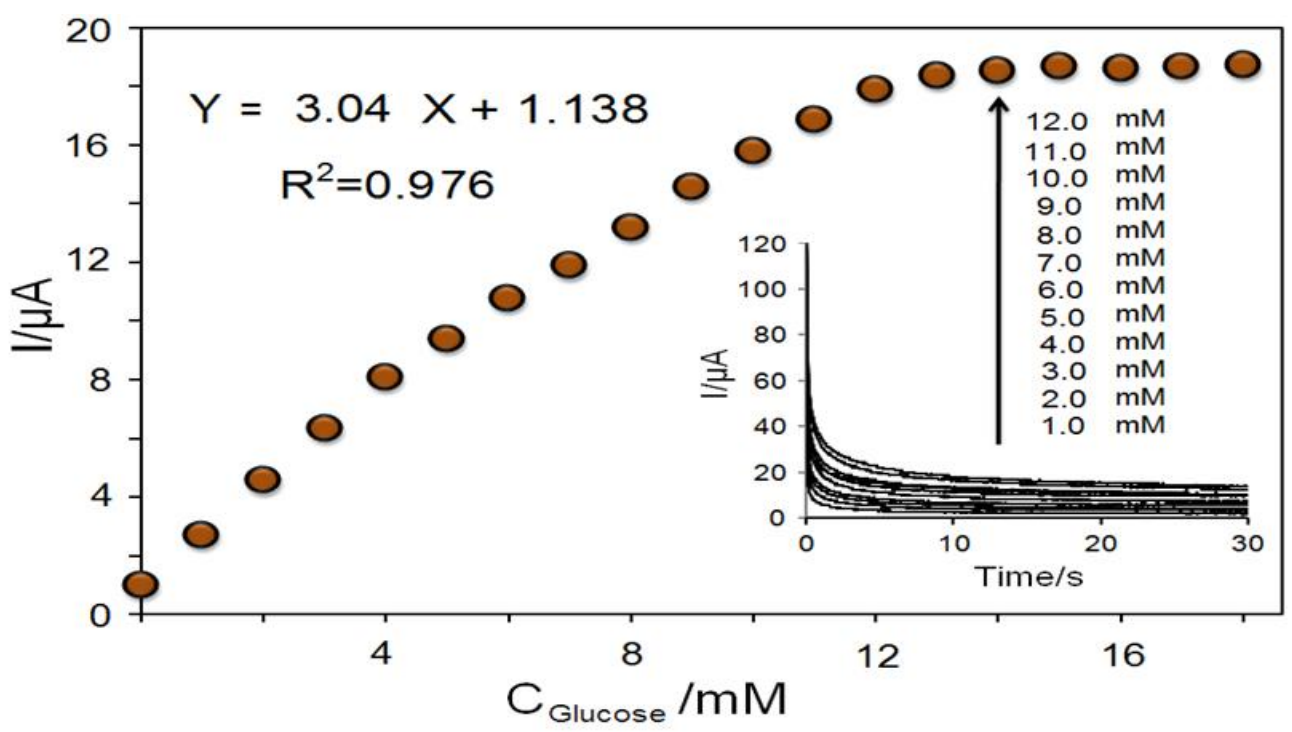

Fig.6. Anodic peak current response (Ipa) vs. glucose concentration at high concentration. (0-18 mM) 
Anti-interference studies are also important and essential for biosensors. Some easily oxidative species, such as ascorbic acid (AA), ethanol, and glycin usually co-exist with glucose in human blood. The interference experiment was carried out by successive injections of interfering species. The amperometric

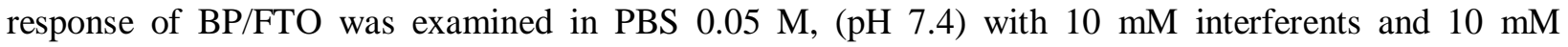
glucose. A well-defined glucose response was obtained (fig.7), and insignificant responses were observed for interfering species and the BP /FTO electrode shows good selectivity for glucose detection.

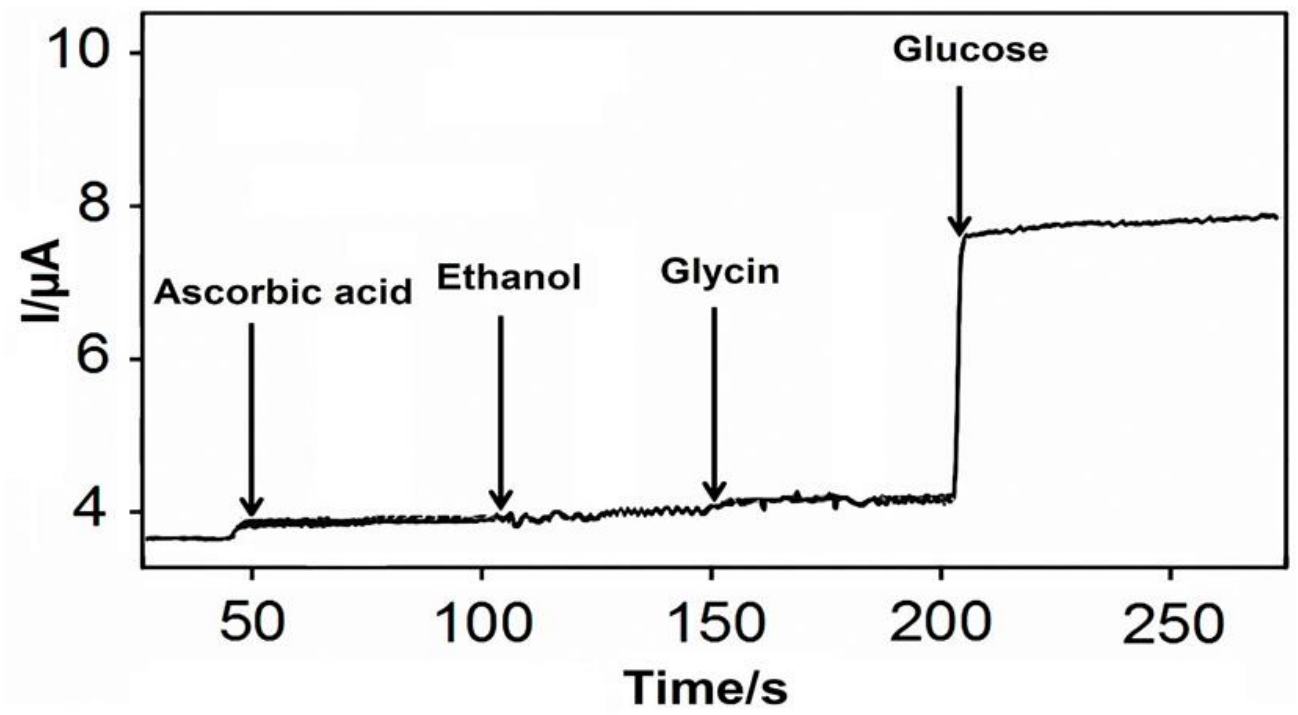

Fig.7. Selectivity of BP /FTO in PBS 0.05 M, (pH 7.4) with $10 \mathrm{mM}$ interferents and $10 \mathrm{mM}$ glucose

The longer term stability is an important parameter for the evaluation of the performance of the biosensor, which was obtained by measuring its current response to glucose within a 28-day period (fig.8). The current response of the BP electrode was approximately $90 \%$ of its original counterpart, which can be mainly attributed to the chemical stability of BP. 


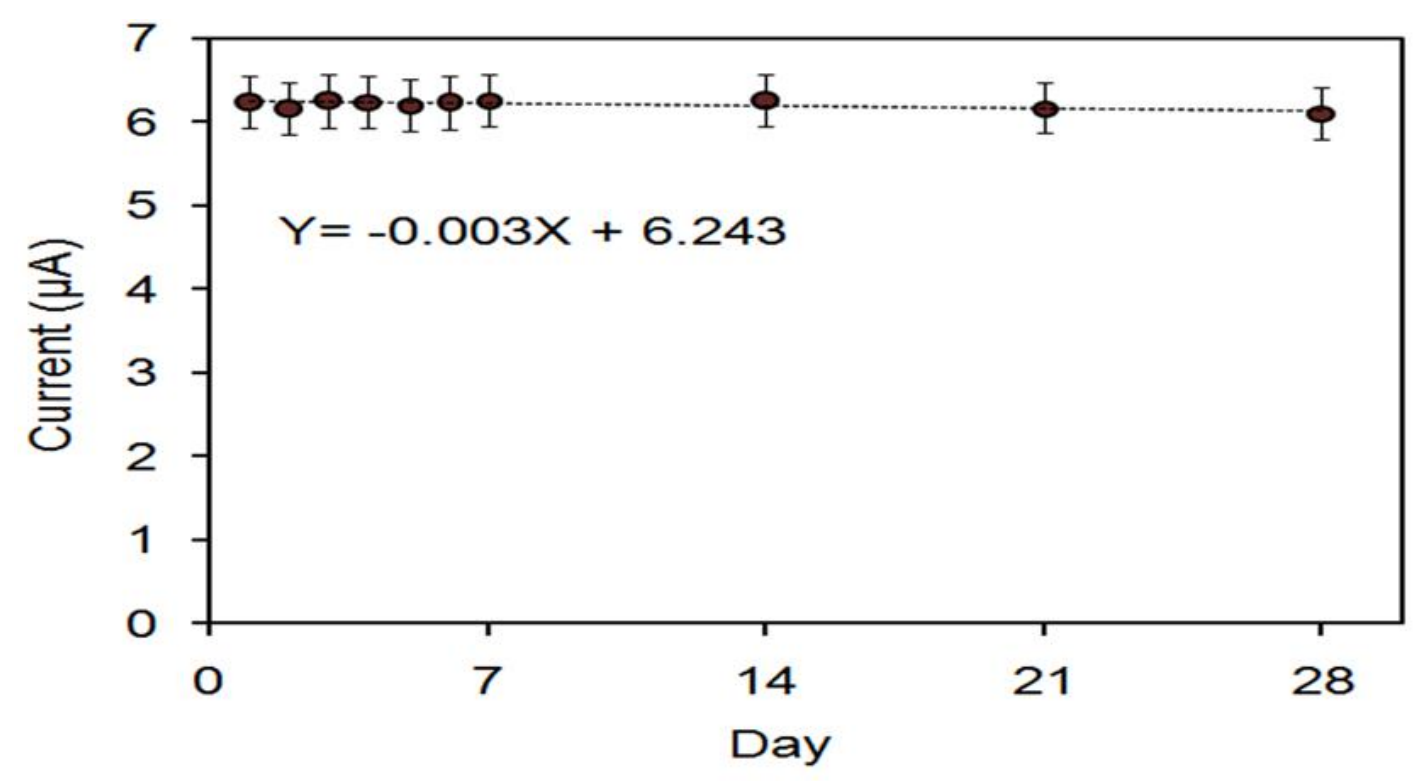

Fig. 8 .Stability response of BP/FTO after 28 days

\section{4: Conclusion:}

We synthesised BPs with five different surfactants in a similar procedure and investigated the effect of the type of surfactants on the surface morphology and electrochemical behaviour of the prepared BPs. Experimental results showed that the type of surfactants had a significant role in the dispersion of SWCNTs and on the quality of BPs. This was consistent with the morphological results of BPs prepared with different surfactants. In this regard, the BP/Tween 20 showed the highest uniformity of surface among the other BPs. Also, the fig. 5 revealed enhanced charge transfer kinetics for BPs prepared by Tween 20 in comparison to others. Also, We have successfully modified electrode by BP. The BP electrode, a novel enzymatic glucose sensor, presents attractive analytical features, such as high sensitivity and stability. The BP electrode can also be used as an amperometric sensor for the routine analysis of glucose in human. Based on the amperometric measurement, it is competitive for the effective determination of glucose with low cost, low liner range, and high sensitivity.

1. Baig, N. and T.A.J.M.A. Saleh, Electrodes modified with 3D graphene composites: a review on methods for preparation, properties and sensing applications. 2018. 185(6): p. 283.

2. Popov, V.N.J.M.S. and E.R. Reports, Carbon nanotubes: properties and application. 2004. 43(3): p. 61-102.

3. Yang, N., et al., Carbon nanotube based biosensors. Sensors and Actuators B: Chemical, 2015. 207: p. 690-715.

4. Lin, Y., et al., Advances toward bioapplications of carbon nanotubes. Journal of Materials Chemistry, 2004. 14(4): p. 527-541. 
5. Bahr, J.L., et al., Functionalization of carbon nanotubes by electrochemical reduction of aryl diazonium salts: a bucky paper electrode. Journal of the American Chemical Society, 2001. 123(27): p. 6536-6542.

6. Gou, J., Single-walled nanotube bucky paper and nanocomposite. Polymer International, 2006. 55(11): p. 1283-1288.

7. Tan, P., et al., A RuO 2 nanoparticle-decorated buckypaper cathode for non-aqueous lithiumoxygen batteries. Journal of Materials Chemistry A, 2015. 3(37): p. 19042-19049.

8. Song, P., C. Liu, and S. Fan, Improving the thermal conductivity of nanocomposites by increasing the length efficiency of loading carbon nanotubes. Applied Physics Letters, 2006. 88(15): p. 153111.

9. Sweetman, L., et al., Synthesis, properties and water permeability of SWNT buckypapers. Journal of Materials Chemistry, 2012. 22(27): p. 13800-13810.

10. Byrne, M.T., C.A. Hanley, and Y.K. Gun'ko, Preparation and properties of buckypaper-gold nanoparticle composites. Journal of Materials Chemistry, 2010. 20(15): p. 2949-2951.

11. Chen, A. and S. Chatterjee, Nanomaterials based electrochemical sensors for biomedical applications. Chemical Society Reviews, 2013. 42(12): p. 5425-5438.

12. Wang, B., et al. Theoretical Prediction and Experimental Study of Mechanical Properties for Random and Magnetically Aligned Single-Walled Carbon Nanotube/Epoxy Composites. in Materials Research Society Symposium Proceedings. 2004. Warrendale, Pa.; Materials Research Society; 1999.

13. Park, J.G., et al., Effects of surfactants and alignment on the physical properties of single-walled carbon nanotube buckypaper. Journal of Applied Physics, 2009. 106(10): p. 104310. 